RESEARCH ARTICLE

\title{
Mycorrhizal colonization in Drosera burmannii Vahl, a Carnivorous Plant: Observation using Light and Fluorescence Microscopy
}

\section{Kripamoy Chakraborty ${ }^{1 *}$, Krishna Talapatra ${ }^{1}$, Aparajita Roy Das², Ajay Krishna Saha', Panna Das ${ }^{1}$}

\footnotetext{
${ }^{1}$ Microbiology Laboratory, Department of Botany, Tripura University, Suryamaninagar 799022, Tripura, India

${ }^{2}$ Mycology and Plant Pathology Laboratory, Department of Botany, Tripura University, Suryamaninagar 799022, Tripura, India
}

Study Area:Suryamaninagar, West Tripura, India Coordinates: $23^{\circ} 45^{\prime} 44.00^{\prime \prime} \mathrm{N} ; 91^{\circ} 15^{\prime} 48.43^{\prime \prime} \mathrm{E}$

Key words: Insectivorous plant, AM fungi, DSE, Microscopy

\section{Introduction:}

Arbuscular mycorrhizal (AM) fungi are probably the most widespread terrestrial symbiosis (Fitter, 2005), formed by $70-90 \%$ of land plant species (Smith \& Read, 2008) with fungi belong to a monophyletic phylum Glomeromycota (Schüßler et al., 2001). The plant furnishes carbon to the fungal partner and in return fungal partner assist the plants in their uptake of phosphorus $(\mathrm{P})$, nitrogen $(\mathrm{N})$ and other mineral nutrients from deficient sites (Smith \& Read, 2008) by extending the soil volumes that ensure the improved plant growth through better nutrition uptake and alleviate toxicity of pollutants (Bolan, 1991; Marschner, 1998).

Mycorrhizal and non-mycorrhizal plant roots are also known to colonized by a diverse group of pigmented, septate and thick walled fungi called dark septate endophyte (DSE) fungi (Sathiyadash et al., 2010) and reported to coupled with enhancement of plant growth, biomass accumulation, nutrient acquisition or fitness of the inoculated seedlings (Rodriguez et al., 2009). Jumpponen \& Trappe (1998) recapitulated the incidence of DSE associations in nearly 6,00o plants species representing about 320 genera and 100 families, but only 59 were tropical plant species. Extensive literature survey revealed that roots of carnivorous plants are nonmycorrhizal (Macdougal, 1899; Juniper et al., 1991; Brundrett, 2009) although, Chambers et al. (2008); Fuchs \&

\section{Abstract}

In the present investigation, the insectivorous plant, Drosera burmannii Vahl inhabiting in the natural ecosystem was evaluated for categorizing the status of mycorrhizal colonization under fluorescence and light microscope. The results revealed the occurrence of dual colonization by AM and DSE fungi in D. burmannii. Mycorrhizal structures such as arbuscules possessed 21.48 $\%$, vesicles having $18.66 \%, 65.56 \%$ of hyphae and $7.14 \%$ of DSE colonization in the root. The colonization by mycorrhizal fungi was noticed in the cortical cells and it was more in the outer cortical cells. Fluorescing vascular bundles and the presence of root hairs were observed. Hyphal coils and hyphal penetration in the epidermis along with fluorescing arbuscules were also observed. The study suggests the presence of mycorrhizal associations may assist benef it to the plant to acquire nutrients from the soil.

Haselwandter (2004) have reported that the plants may occasionally be colonized by facultative mycorrhiza. It is a general notation that carnivorous plants lack AM fungal colonization due to their inimitable nutrients acquisition mode resulting in diminution of AM fungal dependency (Harikumar, 2013).

Drosera burmannii Vahl prevalently known as the Sundews or Surya Shisir belonging to the family Droseraceae is an annual carnivorous herb. It is one of the most commonly occurring carnivorous species among Indian carnivorous plants and found gushing in tropical and subtropical parts of the country. D.burmannii contains naphthoquinones, flavonoids like quercetin and hyperoside (Wagner \& Baldt, 1984; Wang, 1998) and possesses anticonvulsant activity (Hema et al., 1983).

Detection and quantification of AM fungi along with the degree of root colonization in the roots of terrestrial plants are indispensable tools in mycorrhizal research. Staining and microscopic methods not only assure reliable data on the degree of root colonization but also consent visualizes the presence of key structural features such as arbuscules, which are the morphological criteria for defining AM associations (Brundrett, 2004). Ames et al. (1982) and Jabaji-Hare et al. (1984) postulated the quantif ication of the auto fluorescing 'arbuscules' as a nondestructive method for measuring root colonization by AM fungi. An extensive study by Gange et al. (1999) unveiled 


\section{RESEARCH ARTICLE}

the arbuscular colonization intensity in a wide range of plants by staining with Chlorazol black E (CBE), acid fuchsin and Trypan Blue with the level of the auto fluorescing 'arbuscules' and summarized that in most plants the number of autofluorescing 'arbuscules' seemed much higher compared to the number of stained arbuscules. Vierheilig et al. (2001) also observed that autofluorescing collapsed arbuscules are not stained by the dyes commonly used to stain mycorrhizal fungi.

The meager information on the role mycorrhizal fungi in the insectivorous plants inhabiting in natural ecosystem promoted us to evaluate the status of mycorrhizal association in $D$. burmannii under the fluorescence microscope and light microscope.

\section{Materials and methods:}

Study site: sampled using quadrate of $1 \mathrm{~m} \mathrm{x} \mathrm{mm}$, the soil and root sample of the plant was collected from field during March-April, 2016. The plant material was collected from the embankment of rain fed agricultural land where rice was cultivated once a year.

Root sampling: the roots of randomly selected five healthy $D$. burmannii plants were collected and mixed in marked polythene bags and transferred to the laboratory for root processing and estimation of mycorrhizal colonization. Care was taken not to damage the roots during collection. The roots were collected carefully and properly identified in order to avoid the collection of roots from inadvertent species.

Soil sampling for isolation of AM fungi: the soil samples of approximately $500 \mathrm{~g}$ at depths of approximately $0-20 \mathrm{~cm}$ around four points of plants were collected and mixed, labeled and zipped in polythene bags from the selected site and transported to the laboratory for processing. After transferring to the laboratory, the soil samples were kept for the quantification of spore density and AM fungal composition determination.

Assessment of AM Fungal and DSE colonization: the collected fine roots were thoroughly washed in several changes of running tap water to remove the adhering soil particles and organic debris. After adequate washing, the root samples were cut into approximately $1 \mathrm{~cm}$ in size and cleared with $10 \% \mathrm{NaOH}$ at $90^{\circ} \mathrm{C}$. The duration of heating is depending on the clearance of the root samples. The cleared roots were washed again with running tap water for 4-5 times and bleached in 2-3 drops of alkaline $\mathrm{H}_{2} \mathrm{O}_{2}$ with slight heating. The root samples were stained by following the method (Roy Das et al., 2015). After a while, the stained root samples were mounted with lactoglycerol on a greese free glass slide and observed the mycorrhizal structures such as arbuscules, vesicle, septate and aseptate hyphae and microsclerotia under a compound microscope (Olympus $C$ $X$ 2ii) fitted with the camera and software, SImage in the computer. The estimation of AM and DSE fungal
Ambient Science, 2019: Vol. 06(1); 16-20 DOI:10.21276/ambi.2019.06.1.ra02

colonization were done by magnif ied intersection method (McGonigle et al., 1990).

Fluorescence microscopy: the fresh root segments were cleaned of soil with water and cut transversely with the help of sterilized blades and dipped into the $0.1 \% \mathrm{NaOH}$ solution for 10 minutes. After that, the fine root segments were washed several times with tap water followed by distilled water. Then the root segments were stained with Alanine Blue and Ujala fabric whitener and kept it for 30 minutes. After 30 minutes the root segments were taken in glass slides with distilled water and observed under a fluorescence microscope (Leica DM 400o) using the filters adjusted to the wavelength of DAPI.

Isolation and identification of AM fungal species: AM fungal spores were extracted from the rhizospheric soil by wet sieving and decanting method (Gerdeman \& Nicolson, 1963). Intact AM fungal spores were picked up with a needle in glass slides with polyvinyl alcohol-lactoglycerol and observed under a dissecting microscope (Koske \& Tessier, 1983). Taxonomic identification of these spores was done on the basis of spore morphology, subcellular characters, and ornamentation in the spore wall. Spore characters were compared with the original descriptions (www.amfphylogeny.com).

Root morphology study: root hair length and root hair density/cm of root segments were counted from the selected root segments used for quantification of fungal colonization. The three-dimensional counting of root segments was carried out by adjusting the focus of a microscope's plane (Ma et al., 2001).

\section{Results :}

Mycorrhiza of D. burmannii under a light microscope: The plants of $D$. burmannii along with its root structure are depicted in Figure 1 a-d. The root is a coarse structure with mean root hair length is $0.8 \pm 0.004 \mathrm{~mm}$ and root hair density is $3.48 \pm 0.241 \mathrm{~cm}$ of root. The arbuscular mycorrhizal structures viz., intercellular hyphae, vesicles, arbuscules, and septate hyphae were observed in the roots of $D$. burmannii (Fig.- 2). The roots are colonized by aseptate hyphae forming appressoria on the root surface (Fig.- 2a). The presence of vesicles are noticed (Fig.- 2b). Arum type of AM fungal morphology is observed (Fig.- 2d) with arbuscules inside the cells connected with intercellular hyphae running in the intercellular spaces. Dual (AM \& DSE fungi) colonization was observed in $D$. burmannii. The DSE colonization was marked by the conspicuous presence of microsclerotia and septate melanized hyphae in the root sections (Fig.- 2 e \& f).

Mycorrhizal structures such as arbuscules possessed $21.48 \pm 3.43 \%$, vesicles having $18.66 \pm 3.23 \%, 65.56 \pm 1.85 \%$ of hyphae and $7.14 \pm 1.9 \%$ of DSE colonization in the root. A negative insignificant correlation $(\mathrm{R}=-0.07)$ was noted between mycorrhizal colonization and root hair characters. 


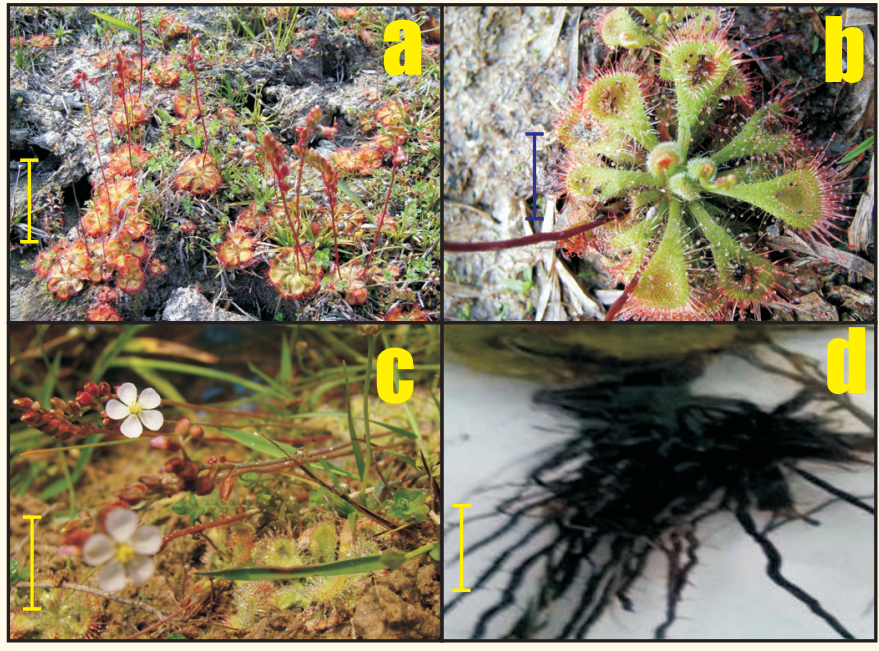

Figure-1: Habitat and root architecture of Drosera burmannii. (a) Habitat. (b) Plant with trapped insect. (c) Flowering twig (d) Root architecture. (Scale bars: $a=1 \mathrm{~cm} ; \mathrm{b}=1.5 \mathrm{~cm} ; \mathrm{c}=2 \mathrm{~cm} \& \mathrm{~d}=1.2 \mathrm{~cm}$ )
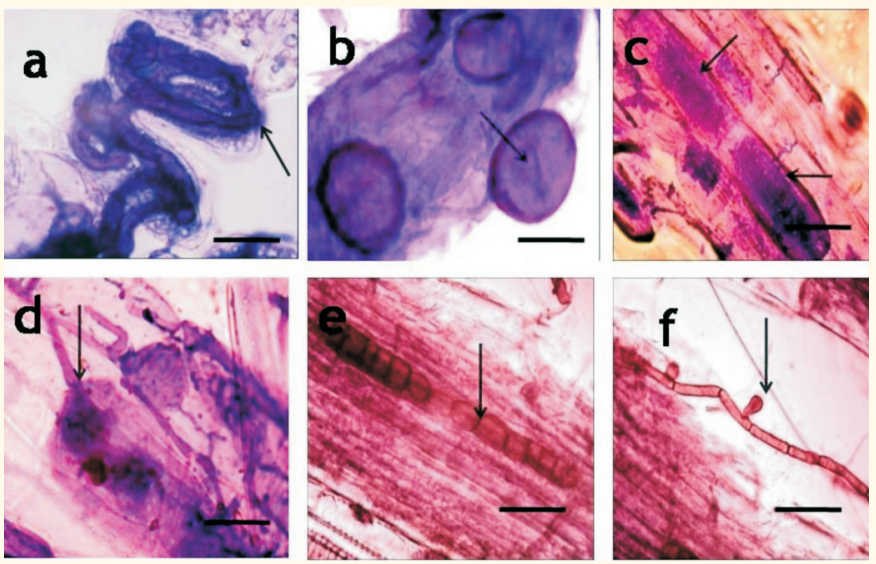

Figure-2: Light microscopy images of AM and DSE fungal colonization in the root segments of D.burmannii.(a) Root segments showing intracellular hyphal coil, (b)Root segments showing intraradical spores in the epidermal layers, (c) Root segments showing Arbuscule (arrow), (d) Root segments showing Arum type morphology, (e) Root segments showing microsclerotia (arrow) of Dark septate endophyte (DSE), (f) Root segments showing melanized and regularly septate hyphae of DSE (arrow). (Scale bars: a,d $=\mathbf{2 0 0} \mu \mathrm{m} ; \mathrm{b}, \mathrm{c}, \mathrm{e} \& \mathrm{f}=\mathbf{1 0 0} \mu \mathrm{m}$ ).

Mycorrhiza of $D$. burmannii under a fluorescence microscope: the colonization by mycorrhizal fungi was noticed in the cortical cells and it was more in the outer cortical cells. A freehand section also showed arbuscules and hyphal coils of the mycorrhizal fungi in the cortical cells. Fluorescing vascular bundles and the presence of root hairs were observed (Fig.- za \& b). Hyphal coils and hyphal penetration in the epidermis along with fluorescing arbuscules were also observed (Fig.-3c \& d).

AM fungal composition: the spore density in the rhizospheric soil inhabiting by $D$. burmannii was $30.33 / 50 \mathrm{~g}$ of soil samples. A total of seven AM fungal morphotypes belonging to three different genera are distinguished on the basis of spore morphology; included Glomus sp.1 to Glomus
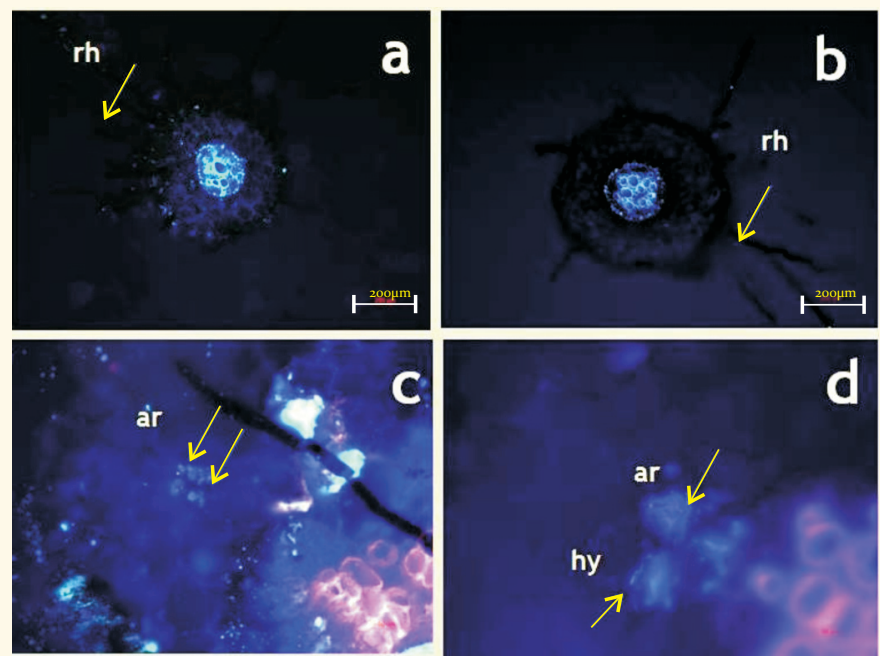

Figure-3: Fluorescence Microscopy images of Mycorrhizal colonization in the root segments of D.burmannii. (a-d) Transverse Section (T.S.) of D.burmannii root stained with Alanine Blue and Ujala observed under Fluorescence Microscope. (a \& b) Fluorescing vascular bundles and presence of root hairs. (c) Arbuscule of AM fungi stained with Alanine Blue (arrow). (d) Arbuscules and hyphal coil stained with Ujala.ar=Arbuscule, hy =Hyphae and $r h=$ Root hairs. (Scale bars: $a-b=200 \mu m ; c-d=50 \mu m$ )
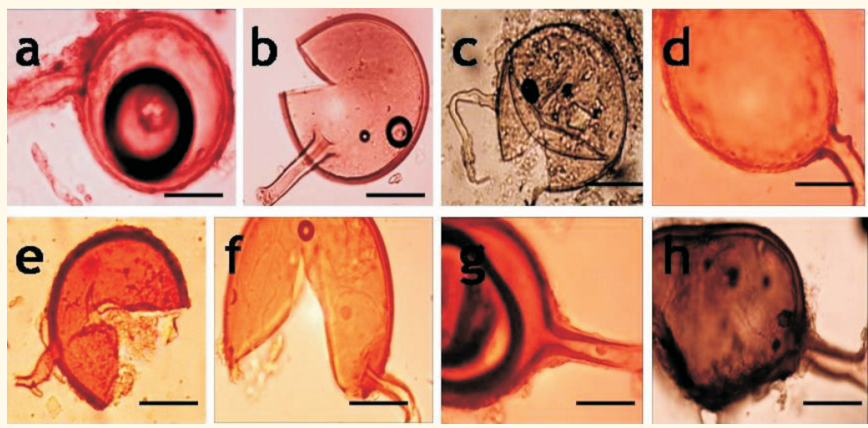

Figure - 4: Arbuscular mycorrhizal fungal spores isolated from the rhizospheric soil of D.burmannii. (a) Glomus sp.1, (b) Glomus sp.2, (c) Paraglomus sp. (d\&g) Rhizophagus diaphanus, (e) Glomus sp.3, (f) Glomus sp.4, (h) Rhizophagus irregularis (Scale bar: $\mathrm{a}-\mathrm{h}=100 \mu \mathrm{m}$ ).

sp.4, Paraglomus sp, Rhizophagus diaphanus and Rhizophagus irregularis as depicted in the Figure- 4.

\section{Discussion:}

The study attributes mycorrhizal colonization status in carnivorous plant D.burmannii. The fungal structures such as aseptate intercellular hyphae, septate hyphae, microsclerotia, vesicles and arbuscules were found in the cortex representing typical mycorrhizal colonization. Vesicles were found to aggregate at certain points in the root cortex in D.burmannii. The incidence of AM fungi with fully-developed fungal structures in Drosera is consistent with the reports of Fuchs \& Haselwandter, (2004) and Weishampel \& Bedford, (2006) who observed the colonization of AM fungi in the roots of D.intermedia and D.rotundifolia. Harikumar (2013) also recorded AM fungal 


\section{RESEARCH ARTICLE}

colonization in the roots of D.burmannii and D.indica from India. AM morphology in Drosera corresponded to the Arum-type as reported earlier (Harikumar, 2013). Morphological types of AM fungi have been examined in many plant taxa (Dickson, 2004; Matsuda et al., 2005; Muthukumar \& Prakash, 2009; Das \& Kayang, 2010; Das et al., 2013; Chakraborty et al., 2016) although physiological or functional disparity between Arum-type and Paris-types has not yet been fully elucidated. However, this is the first report of dual colonization specifically by AM and DSE fungi in D.burmannii. The AM fungal colonization in roots of D.burmannii was much higher than the earlier study (Harikumar, 2013). Detection by the use of light and fluorescence microscopy also revealed that the roots of D.burmannii is colonized by AM and DSE fungi. Glomus was highly abundant in the soil inhabited by D.burmannii. The possible reason for Glomus abundance is rather complex though factors such as adaptability to acid soils, high competitiveness and/or reproductive capacity (Sieverding et al., 1991) may be contributing to this. The findings of earlier workers concerning the AM fungal composition in the rhizosphere of Indian soils revealed that Glomus is the dominant genus (Muthukumar \& Udaiyan, 2000; Bhattacharya \& Bagyaraj, 2002). The occurrences of Glomus from Tripura, Northeast India was also found in the earlier studies (Debnath et al., 2015, 2017; Chakraborty et al., 2016; Sinha et al., 2016).

Extensive research is required to unknot the exact role of the AM symbiosis and the stages of the plant growth influenced by such symbiosis. The study points out the presence of mycorrhiza which may serve as another way of availing nutrients from the soil.

\section{Acknowledgements:}

The authors are thankful to the Head, Department of Botany, Tripura University for providing the laboratory facilities. KC is grateful to the Department of Science and Technology, Government of India for the INSPIRE fellowship. Authors are thankful to the Co-ordinator, State Biotech Hub, Tripura University for using the fluorescence microscope..

\section{References:}

Ames, R.N., Ingham, E.R. \& Reid, C.P.P. (1982): Ultraviolet induced autofluorescence of arbuscular mycorrhizal root infections: an alternative to clearing and staining methods for assessing infections. Can. J. Microbiol., 28(3):35-355.

Bhattacharya, S. \& Bagyaraj, D.J. (2002): Arbuscular mycorrhizal fungi associated with Coffea arabica. Geobios., 29:93-96.

Bolan, N.S. (1991): A critical review on the role of mycorrhizal fungi in the uptake of phosphorus by plants. Plant Soil, 134(2):189207.

Brundrett, M.C.(2004): Diversity and classif ication of mycorrhizal associations. Biol Rev Camb Philos Soc. 79(3): 473-495.

Brundrett, M.C. (2009): Mycorrhizal associations and other means of nutrition of vascular plants: understanding the global diversity of host plants by resolving conflicting information and developing reliable means of diagnosis. Plant Soil.,
Ambient Science, 2019: Vol. 06(1); 16-20 DOI:10.21276/ambi.2019.06.1.ra02

320(1-2):37-77.

Chakraborty, K., Sinha, S., Debnath, A., Roy Das, A., Saha, A.K., \& Das, P. (2016): Arbuscular mycorrhizal fungal colonization in three different age groups of rubber plantations in Tripura, North-East India. Plant Path. Quarantine, 6(2):122-131..

Chambers, S.M., Curlevski, N.J. \& Cairney, J.W. (2008): Ericoid mycorrhizal fungi are common root inhabitants of nonEricaceae plants in a south-eastern Australian sclerophyll forest. FEMSMicrobiol. Ecol., 65(2):263-270.

Das, P. \& Kayang, H. (2010): Mycorrhizal colonization and distribution of arbuscular mycorrhizal fungi associated with Michelia champaca L. under plantation system in northeast India. J. Forestry Res., 21(2):137-142.

Das, P., Saha, A.K. \& Kayang, H. (2013): Arbuscular mycorrhizal fungal morphology in Michelia champaca L. J. Mycopathol Res., 51(1):177-179.

Debnath, A., Karmakar, P., Debnath, S. , Roy Das, A., Saha, A.K. \& Das, P.(2015): Arbuscular mycorrhizal and dark septate endophyte fungal association in some plants of Tripura, North-East India. Current Res. Environ. Appl. Mycol., 5(4): 398-407.

Debnath, A., Sinha, S., Talapatra, K. Chakraborty, K. Saha, A.K. \& Das, P. (2017): Comparison of arbuscular mycorrhizal colonization and diversity of two different rubber plantations of Tripura, northeast India, 239- 247 pp. In: Sinha, R. \& Sinha, R.K. (eds.) Trends in Frontal Areas of Plant Science Research. Pub. by: Narosa Publishing House, India.

Dickson, S. (2004): The Arum- Paris continuum of mycorrhizal symbioses. New Phytologist, 163(1):187-200.

Fitter, A. H. (2005): Darkness visible: reflections on underground ecology.J.Ecol., 93(2):231-234.

Fuchs, B. \& Haselwandter K. 2004: Red list plants: colonization by arbuscular mycorrhizal fungi and dark septate endophytes. Mycorrhiza., 14(4):277-281.

Gange, A.C., Bower, E., Stagg, P.G., Aplin, D.M. Gillam, A.E. \& Bracken, M. (1999): A comparison of visualisation techniques for recording arbuscular mycorrhizal colonisation. New Phytol., 142(1):123-132

Gerdemann, J. W. \& Nicolson, T.W. (1963): Spores of mycorrhizal endogone species extracted from soil by wet-sieving and decanting method. Transac. Brit. Mycol. Soc., 46(2):235-244.

Harikumar, V.S. (2013): Are there arbuscular mycorrhizal associations in carnivorous plants Drosera burmanii and $D$. indica? Botanica Servica., 37(1):13-19.

Hema, B., Bhupendra, S., Mohamed Saleem, T.S. \& Gauthaman, K. (2009): Anticonvulsant effect of Drosera burmannii Vahl. Int. J. Appl. Res. Nat. Prod., 2(3):1-4.

Jabaji-Hare, S.H., Perumalla, C.J. \& Kendrick, W.B. (1984): Autofluorescence of vesicles, arbuscules, and intercellular hyphae of a vesicular-arbuscular fungus in leek (Allium porrum) roots. Can. J Bot., 62(12):2665-2669.

Jumpponen, A. \& Trappe, J.M. (1998): Dark septate endophytes: a review of facultative biotrophic root colonizing fungi. New Phytol ., 140(2):295-310.

Juniper, B.E. \& Robins, R.J. \& Joel, D.M. (1991): Carnivorous plants. Kew Bull., 46(1):179-182.

Koske, R.E., \& Tessier, B. (1983): A convenient, permanent slide mounting medium. Mycol. Soc. Am. Newsl., 34:59 
Ma, Z., Bielenberg, D.G., Brown, K.M. \& Lynch, J.P. (2001): Regulation of root hair density by phosphorus availability in Arabidopsis thaliana. Plants, Cell Environ., 24(4):459-467.

Macdougal, D.T. (1899): Symbiosis and saprophytism. Bull. Torrey Bot. Club, 26(10):511-530.

Marschner, H. (1998): Role of root growth, arbuscular mycorrhiza and root exudates for the efficiency in nutrient acquisition. Field Crop Res.. 56(1-2):203-207.

Matsuda, Y., Murahashi, F., Nakanishi, K., \& Ito, S-i. \& Kimoto, M., (2005): Arbuscular mycorrhizas on Athyrium yokoscense and A.niponicum grown at a lead-contaminated site. Mycoscience, 46(4):261-264.

McGonigle, T.P., Miller, M.H., Evans, D.G., Fairchild, G.L., \& Swan, J.A.(1990): A new method which gives an objective measure of colonization of roots by vesicular-arbuscular mycorrhizal fungi. New Phytol., 115(3):495-501.

Muthukumar, T. \& Udaiyan, K. (2000): Arbuscular mycorrhizas of plants growing in Western Ghats region, Southern India. Mycorrhiza, 9(6):297-313.

Muthukumar, T. \& Prakash, S. (2009): Arbuscular mycorrhizal morphology in crops and associated weeds in tropical agroecosystems. Mycoscience, 50(3):233-239.

Rodriguez, R.J., White, F.A., Arnold, A.E. \& Redman, R.S. (2009): Fungal endophytes: diversity and functional roles. New Phytol.,182(2):314-330.

Roy Das, A., Talapatra, K., Chakraborty. K., Saha, A.K., \& Das, P. (2015): A commercially available fabric whitener serves as a stain for detection of arbuscular mycorrhizal fungi in roots. Mycorrhiza News., 27(3):2-4.
Sathiyadash, K., Muthukumar, T. \& Uma, E. (2010): Arbuscular mycorrhizal and dark septate endophyte fungal associations in South Indian grasses. Symbiosis, 52(1):21-32.

Schüßler, A., Schwarzott, D. \& Walker, C. (2001): A new fungal phylum, the Glomeromycota: phylogeny and evolution. Mycol. Res., 105(12):1413-1421

Sieverding, E., Friedrichsen, J. \& Suden, W.(1991): Vesicular arbuscular mycorrhiza management in tropical agroecosystems. Deutsche Gesellscraft fur Technische Zusammenarbeit, Bremer, Germany.

Sinha, S., Chakraborty, K., Saha, A.K.\& Das, P.(2016): Mycorrhizal colonization in plants from selected home garden of Tripura. L. Mycopathol Res., 54(3):349-353.

Smith, S.E. \& Read, D.J. (2008): Mycorrhizal Symbiosis. Pub. by:Academic Press London. 800 p.

Vierheilig, H., Knoblauch, M., Juergensen, K., van Bel, A.J.E., Grundler, F.M.W. \& Piche', Y. (2001): Imaging arbuscular mycorrhizal structures in living roots of Nicotiana tabacum by light, epifluorescence and confocal laser scanning microscopy. Can. J. Bot., 79(2):231-237.

Wagner, H. \& Baldt, S. (1984): Plant Drug Analysis. Pub. by: Springer-Verlag Berlin, Heidelberg, New York, Tokyo. 369p.

Wang, Q., Su, J. \& Zeng. (1998). The isolation and identification of flavonoids from Drosera burmannii. Zhong Yao Cai., 21(8):401-403.

Weishampel, P.A. \& Bedford, B.L. ( 2006): Wetland dicots and monocots differ in colonization by arbuscular mycorrhizal fungi and dark septate endophytes. Mycorrhiza., 16(7):495502. 